\title{
Wearable Control Using Feedforward Neural Networks has Been used to Control the Manipulator Arm of Field and Service Robots
}

\author{
Archit Fadhilah $^{1}$, J D Setiawan ${ }^{1}$, M Munadi $^{1}$ and M Ariyanto ${ }^{1}$ \\ \{Architfadhilah@undip.ac.id\} \\ ${ }^{1}$ Mechanical engineering Department, Diponegoro University, Semarang, Indonesia
}

\begin{abstract}
Controlling the manipulator arm of a Field and Service Robot (FSR) using joystick input is a time-consuming and wasteful process. Operators who must control correctly and quickly must undergo a specialized training program and devote a considerable amount of study time. In this study, wearable control devices were used to replace joysticks, and Artificial Neural Networks were employed to run the wearable control devices. Wearable control devices were used to replace joysticks in this study (ANN). The Myo Armband, which is worn on the right arm of the operator, is the wearable control device that was used in this experiment. This sensor is composed of a variety of components, including an electromyography sensor (EMG), a 3-axis accelerometer, and a 3-axis gyroscope, among others. Whenever the operator moves his or her arm, the command to move the 3D Manipulator Arm in MATLAB / Simulink will be dependent on the operator's arm and the position of the operator's arm inside the workspace. By using the Inertial Measurement Unit (IMU) sensor that is included into the Myo armband, it is possible to detect and show the location of the operator's arm and arm position. The ANN method is used in conjunction with the IMU to handle data received from the sensor. Feedforward Neural Network (FNN) processing is used. When the FNN output is used to drive the 3D Manipulator Arm model in SimMechanics, the user/operator arm angle is controlled by the user/operator arm angle, which is controlled by the FNN output. It has been discovered that utilizing the FNN regression technique, it is feasible to successfully control a $3 \mathrm{D}$ animation of the manipulator arm in real time using the manipulator arm. This results in an average accuracy value of 0.973 for R derived from training, validation and testing, as well as an overall accuracy value of 0.973 . Since all figures are more than 0.950 , this shows that the neural network works very well.
\end{abstract}

Keywords: Wearable control, Myo armband, artificial neural network, manipulator arm.

\section{Introduction}

It is abbreviated as FSR (field and service robot), and it is a robot that is intended to help people in their jobs, whether they are inside or outside of the room. In a less secure workplace or work location, FSR may be called upon to perform the function of a human guard. A wireless control system is provided for operating the FSR from a remote location. FSR is often fitted with a manipulator arm, which allows it to carry out its responsibilities. Because it is operated by a joystick rather than by hand, this manipulator arm is more difficult and less efficient to use when handled by the operator than when handled by others. Operators must go through specific training and practice for a long period of time before they can become proficient in the operation of manipulator arms. In this research, wearable control is used to take the role of the joystick as the main input and output mechanism. When worn by operators, wearable control is expected to be more convenient and efficient than traditional controls. Wearable control refers to a control device that is worn on the operator's body, such as on the hands, arms, legs, thighs, head, and other limbs, in order to control the operator's bodily movements. In this research, the myo 
armband was used as a wearable control device, and it was very effective. The Myo armband is worn by the operator on his or her right forearm. It is made up of electromyography (EMG) sensors, an inertial measurement unit, and other components, among other things (IMU). It has been shown in study that the Myo armband can be used to measure arm movements [1, which has been published]. In order to operate various devices, the EMG often makes use of the Myo armband [2,3]. EMG has been utilized as a controller for manipulators [2] and [3], and it is one of the methods that has been used. The neural network has been incorporated into the previous control systems [4] [5] and is now part of the system. [6] describes how to simulate the system, which will be used in this research. The simulation will be done in MATLAB. During the simulation of systems, real-time system testing is performed [7]. If an operator or user makes an arm movement, the movement and rotation angle of that arm are utilized to command the movement and rotation angle of the manipulator arm in a similar way, according to this study. It was developed in MATLAB Simscape to build the three-dimensional model of the manipulator arm. The Myo armband's inertial measurement unit (IMU) sensor detects and records the movement and position of the operator's arm. Artificial neural network (ANN) regression, in combination with feedforward neural network (FNN) technique, is required to forward and analyze the data from the inertial measurement unit (IMU). It is possible to use the output of the FNN to control a 3D manipulator arm model that has been built in the MATLAB/Simulink environment..

\section{IMU From Myo Armband}

An armband-style wearable controller known as the Myo armband was used in this study. A wearable sensor, such as the Myo armband, which is manufactured by Thalmic Labs and seen in motion in Figure 1, is an example of a wearable sensor. In order to provide support and protection, it is a one-of-a-kind arm bracelet that the user wears on his or her arm. The Myo armband has three distinct kinds of sensors: an electromyography (EMG) sensor, a 3-axis accelerometer, and a 3-axis gyrometer. The electromyography sensor is the most sensitive of the three sensors. This research takes use of the inertial measurement unit (IMU) of the Myo armband, which is used to produce the signals necessary for directing the movements of a 3D virtual manipulator arm in three dimensions in three dimensions. A total of three acceleration axes and three gyroscope axes are included inside each IMU sensor, and each of these sensors is used in the sensor's design. A depiction of the sensor that was utilized in this research, as well as other information, may be seen in Figure 1.

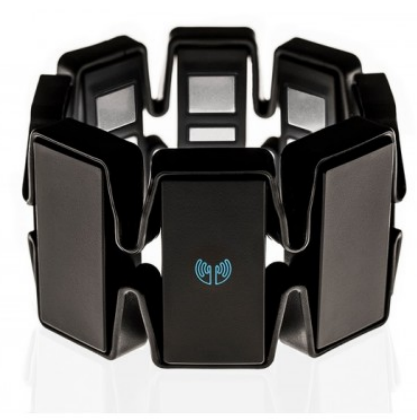

Fig. 1. Myo Armband. 
In order to accurately measure the acceleration of an operator's arm translational acceleration motion, the accelerometer is used in conjunction with the $\mathrm{x}, \mathrm{y}$, and $\mathrm{z}$ axis translational acceleration motion. The Gyroscope's most fundamental purpose is to measure and record the angular velocity of the operator's rotating arm in relation to the rotating axes $\mathrm{x}, \mathrm{y}$, and $\mathrm{z}$, which is the most basic function. The figure below illustrates the movement of the accelerometer and gyroscope in terms of direction and axis.. Fig. 2.

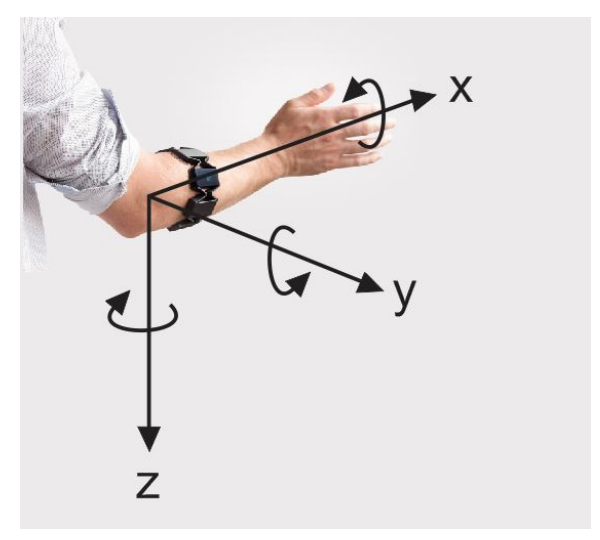

Fig. 2. The Proposed coordinate of accelerometer and gyroscope in Myo armband.

In order to give precise location, the data collected by the accelerometer and gyroscope in a Myo armband is converted into three basic angular movements, which are roll, pitch, and yaw. It is necessary to estimate the roll, pitch, and yaw of the Myo armband using the accelerometer rate gyro (ARG) technique, which is based on three-axis acceleration and three-axis angular velocity data received from the Myo wristband. [8] provides more information on the ARG method for computing roll, pitch, and yaw angles, as well as other related topics. The ARG method will be developed in the MATLAB/Simulink environment, it is anticipated. As shown in Fig. 3, this processing method is carried out inside the MATLAB/Simulink environment. The findings of this research are derived only from the outputs of the roll and pitch angle sensors, respectively. In the Neural Network regression, the roll and pitch will be used as control inputs for the Neural Network, which will be trained using the data from the previous experiment..

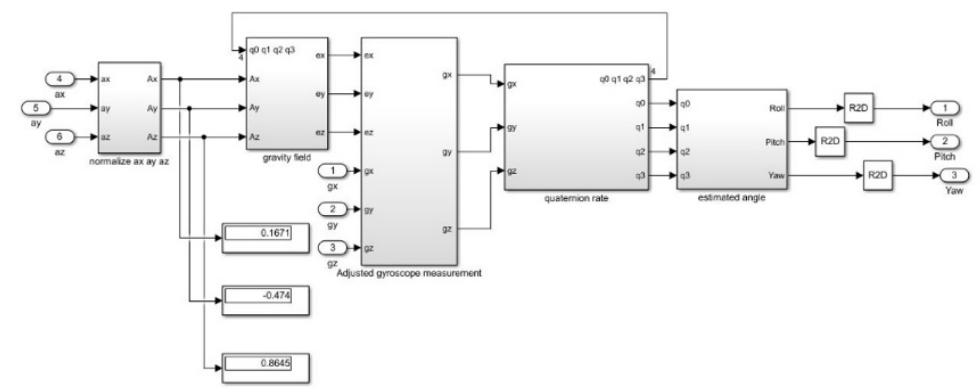

Figure 3. ARG method for roll, pitch, and yaw estimation.

\section{Artificial Neural Network}


When an input signal from a myo armband is received, this model converts it into an output signal in the form of a command to operate a 3D arm manipulator model. It was the FNN artificial neural network that was used in this research. In this instance, the FNN receives two inputs: roll and pitch, which are combined. It also has five hidden layers and four joint angle outputs, which are indicated by the letters A1, A2, a3, and a4. It also has five hidden layers and four joint angle outputs. Four of these outputs are used as control inputs for the threedimensional manipulator arm model, which also includes four of these outputs. The general structure of the FNN Simulink model is shown in FIGURE 4 (see below). However, the data for both the input and the output may be seen in Fig. 5, which depicts both.

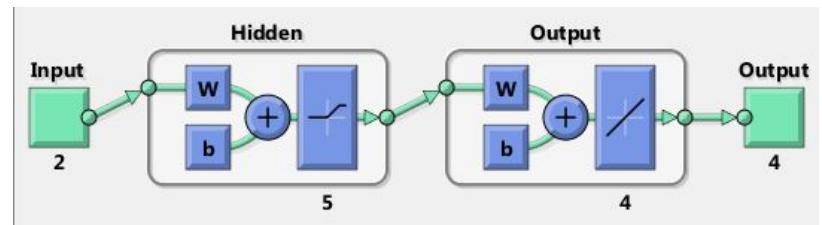

Fig. 4. FNN structure at MATLAB Simulink.

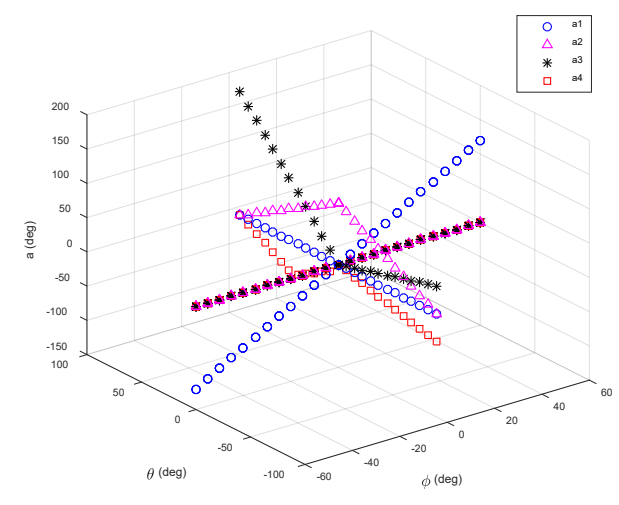

Figure 5 shows the data that was utilized for both input and output.

There are three variations of roll and pitch input that have an effect on the outputs a1 through a4; these three variants are used in conjunction with the results of 225 data points to retrieve the information. The three possible options are as follows: When the pitch has a value, the roll has a value, and when both the pitch and the roll have values, the roll has a value and the pitch has a value and the pitch and roll both have values The entire data set of 225 data points is divided into three subsets: training methods account for $70 \%$ of the total data, validation procedures account for $15 \%$ of the total data, and testing procedures account for $15 \%$ of the total data. It is necessary to utilize the Levenberg-Marquardt Backpropagation method in order to train the FNN for regression, which is a kind of neural network, in order to get good results. Figure 6 depicts the performance of the neural network in the context of regression. The FNN has achieved its convergence value after 15 epochs of computation. The best MSE value that may be achieved as a result of this is 199,078 . After 15 epochs, it reaches its greatest value at this point in time. 


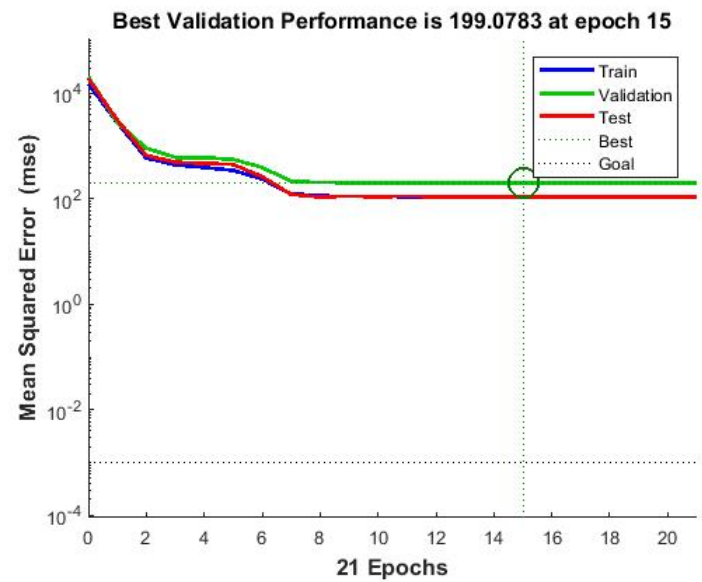

Fig. 6. FNN Performance during training.

It is shown by the Receiver Operating Characteristic (ROC) curve, and it shows how precise the FNN results were achieved. This curve represents the accuracy of the test by plotting the false positive percentage (fp) against the true positive proportion (tp). The receiver operating characteristic curve (ROC curve) represents the accuracy of a test by plotting the false positive percentage (fp) against the true positive proportion (tp). When training, validating, and testing are carried out, $\mathrm{R}$ is $0.973,0.967$, and 0.982 , with a total $\mathrm{R}$ of 0.973 percent as shown in the table.. 7.
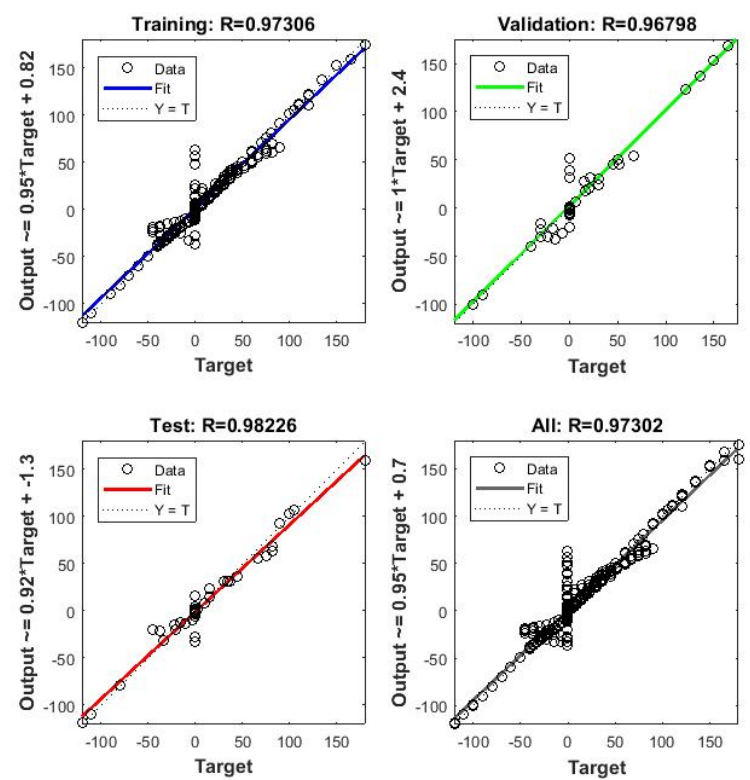

Fig. 7. ROC curve of FNN. 


\section{3D Arm Manipulator Model}

As shown in Fig. 8, the 3D CAD model of the manipulator arm is comprised of a single base, four pivots, and four joints, with a total of eight joints. Angles A1, A2, A3, and A4 are the letters used to indicate the four joint angles. Later, the $3 \mathrm{D}$ computer-aided design (CAD) is exported to SimMechanics 2nd generation, which is a programming environment that operates in the MATLAB/Simulink environment. Exported block diagrams are shown in Figure 9, which shows the diagram that was exported. When using the a1, a2, a3, and a4 functions, the angle movements are measured in degrees and are used as inputs. Image 8. Three-dimensional animation of a manipulator arm from different angles is shown in Figure 8. Revolute joints are used to represent the joint angles of the manipulator arm's joints in the SimMechanics block diagram, which can be seen here.

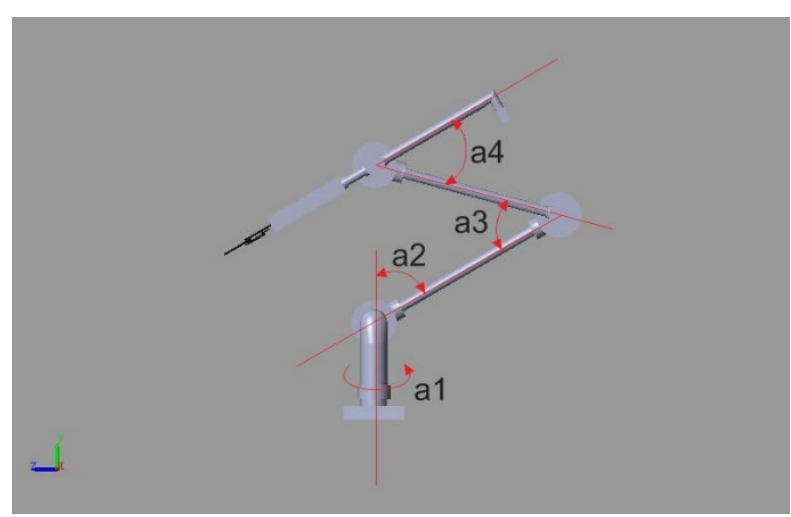

Fig. 8. 3D Arm manipulator model in SimMechanics 3D animation.

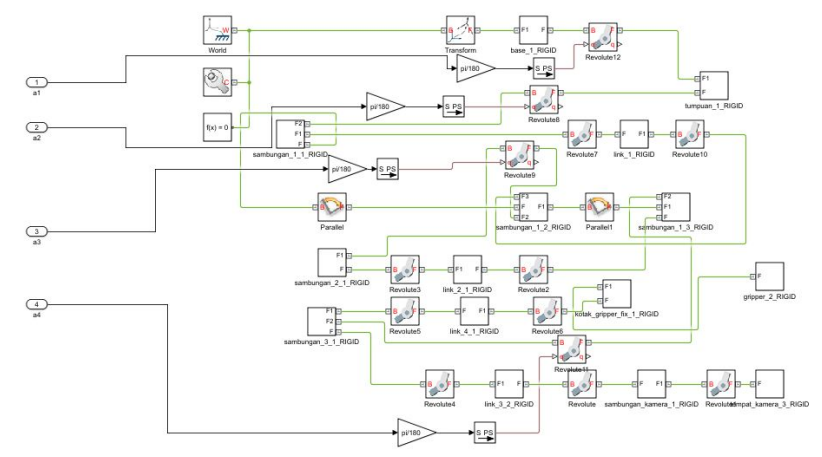

Fig. 9. Simscape 3D manipulator arm in Simulink.

\section{Experimental Work}

In this study, the operator/user wears a Myo wristband on his right arm, which acts as a sensor. In Fig. 10, the pitch angle movement is accomplished by moving the arm up and down, and the roll motion is accomplished by rotating the arm around its $\mathrm{x}$-axis, which is illustrated in Fig. 11 . 


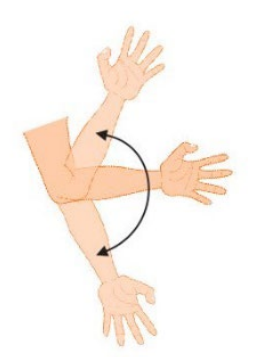

Fig. 10. Arm pitch movement.

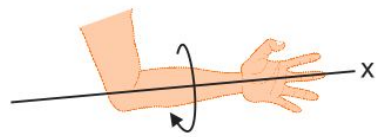

Fig. 11. Arm roll movement.

On the other hand, in Figure 12, you can see the whole MATLAB Simulink system in action. This system is capable of operating in real time. Every movement made by the operator will cause the 3D arm manipulator model to move in the manner shown in Fig. 13.

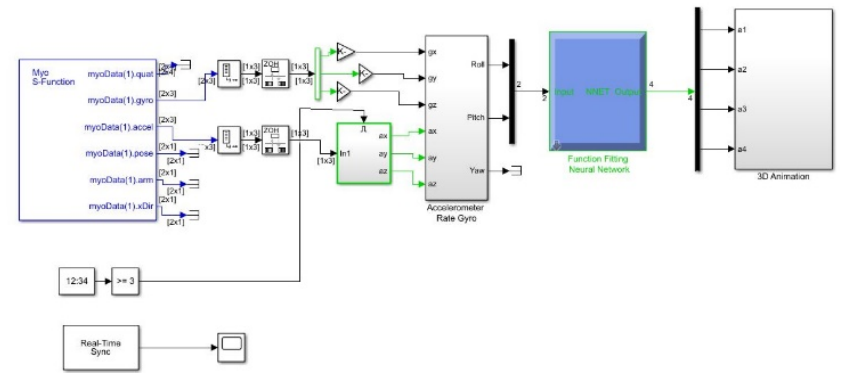

Fig. 12. Overall block diagram of neural network with Myo armband and SimMechanics 3D Animation.
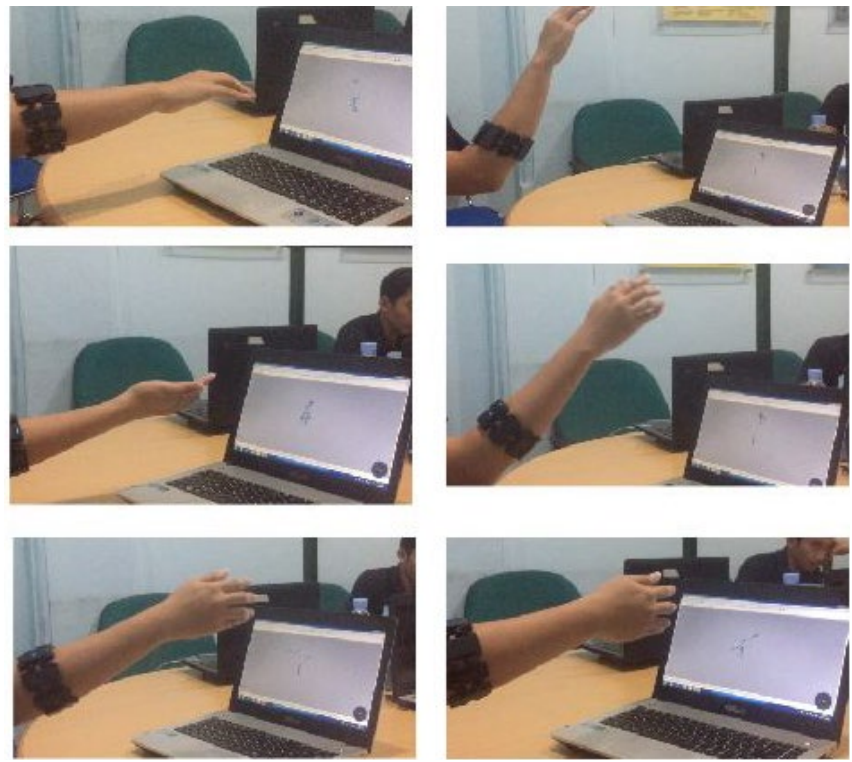
Figure 13. Test result for control 3D arm manipulator model using Myo armband.

During this test, the user's arm is rotated in pitch motion from zero degrees to about 40 degrees, and then it is turned in roll motion from zero degrees to approximately 40 degrees. A diagram of the angle inputs used in the test is given in Fig. 14. The output angles of a2, a3, and a4 that are produced by the trained neural network are shown in Fig. 15 as a function of time..
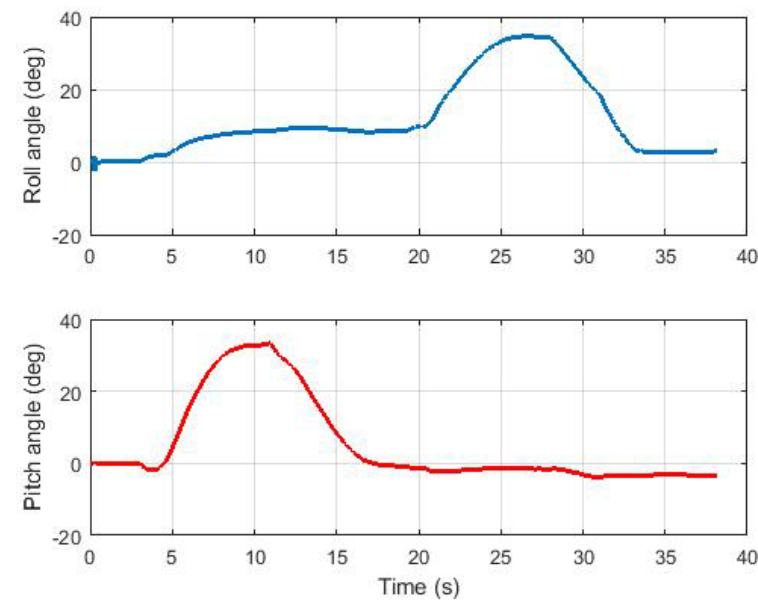

Figure 14. Input FNN Roll and Pitch.

\section{Conclusion and Future Reasearch}

The accuracy of feedforward neural network regression (R) achieved during training (0.973), validation (0.967), and testing (0.982), and total R (0.973), confirm the ROC findings. The network's accuracy was $0.973,0.967$, and 0.973 when trained, verified, and tested. For example, in Figure 12, a 3D manipulator arm model in MATLAB simscape can both visibly and audibly track the operator's arm movement.

\section{Acknowledgments}

This research was financially supported by The Faculty of Engineering, Diponegoro University, Indonesia through Excellent Research Grant 2019.

\section{References}

[1] Hyo Jin Kim, Ye Sol Lee and Donghan Kim Arm 2017 Motion Estimation Algorithm using Myo Armband IEEE International Conference on Robotic Computing 
[2] U. M. Paula and J. M. Robinson 2016 Individual Robotic Arms Manipulator Control Employing Electromyographic Signals Acquired by Myo Armbands Vol. I, ISSN 09734562 India: Research Publications pp. 11241-11249.

[3] Osamu Fukuda, Toshio Tsuji 2003 A Human-Assisting Manipulator Teleoperated by EMG Signals and Arm Motions IEEE Transaction on Robotics and Automation Vol. 19

[4] M. Hajek 2005 Neural Network University of KwaZulu-Natal

[5] I. Rifky, M. Ariyanto and W. Caesarendra 2016 Speech Control of Robotic Hand Augmented with 3D Animation Using Neural Network IEEE EMBS Conference on Biomedical Engineering and Sciences (IECBES)

[6] L.A. Zuniga, J.C. Pedraza, E. Gorrostieta, M.A. Aceves, J.M. Ramos, J.M. Herrera, C.A. Olmos. 2009 Modeling and Simulation of Mechatronic Unit EOD/IEDD 14th International Conference on Method and Models in Automation and Robotics

[7] Min Kyu Kim, Kwang Hyun Ryu, Yong Hwan Oh, Sang Rok Oh and Kee Hoon Kim. 2014 Implementation of Real-Time Motion and Force Capturing System for Telemanipulation based on sEMG Signals and IMU Motion Data IEEE ICRA

[8] Ariyanto, M., 2015. Sensor Fusion using Accelerometer Rate Gyro (ARG) to Estimate Euler Angle of Flight Vehicle (in Indonesian). ROTASI, 17(2), pp.84-92. 